\title{
TOWARD HYBRID TEAMS: A PLATFORM TO UNDERSTAND HUMAN-COMPUTER COLLABORATION DURING THE DESIGN OF COMPLEX ENGINEERED SYSTEMS
}

\author{
B. Song ${ }^{1}$, N. F. Soria Zurita ${ }^{1,2}$, G. Zhang ${ }^{3}$, G. Stump ${ }^{1}$, C. Balon ${ }^{1}$, S. W. Miller ${ }^{1}$, \\ M. Yukish ${ }^{1}$, J. Cagan ${ }^{3}$ and C. McComb ${ }^{1, \bigotimes}$ \\ ${ }^{1}$ The Pennsylvania State University, United States of America, ${ }^{2}$ Universidad San Francisco de Quito, Ecuador, \\ ${ }^{3}$ Carnegie Mellon University, United States of America \\ $\triangle$ uum209@psu.edu
}

\begin{abstract}
Human-computer hybrid teams can meet challenges in designing complex engineered systems. However, the understanding of interaction in the hybrid teams is lacking. We review the literature and identify four key attributes to construct design research platforms that support multi-phase design, hybrid teams, multiple design scenarios, and data logging. Then, we introduce a platform for unmanned aerial vehicle (UAV) design embodying these attributes. With the platform, experiments can be conducted to study how designers and intelligent computational agents interact, support, and impact each other.
\end{abstract}

Keywords: collaborative design, artificial intelligence (Al), design teams, research methodologies and methods

\section{Introduction}

The design of complex engineered systems usually involves coupled design parameters and interrelated design phases, which make such tasks challenging. Computational design tools assist designers in dealing with the complexity of such problems by enabling easier and broader solution search within a design space and reducing the corresponding search cost. Besides being used for design modelling, representation, and simulation, the computational tools have also been increasingly employed for design abstraction and reasoning. In contrast to computational tools, human designers take the initiative to employ much broader exploration strategies and show more intelligence in problem formation, design space understanding, and complicated decision-making (Cooper et al., 2010; Simpson and Martins, 2011; Zhang et al., 2012). Because of this complementary nature, recent work has begun to study human-computer hybrid design teams which integrate and make better use of the strength of both humans and computers for solving design problems (Kollat and Reed, 2007; Simpson et al., 2011). In such a hybrid design team, collaboration and interactions between human designers and computational agents (referred to as intelligent agents in this paper), as well as the composition and characteristics of the team, can influence designers' cognitive processes, exploration strategies, and design outcomes. Consequently, there is a need to study the influence of these factors of human-computer collaboration in designing complex engineered systems.

In practice, most human-computer collaborative design studies rely on design research platforms. These are often software applications through which designers or intelligent agents can build, 
simulate, and analyse their design solution with features that facilitate research data collection. In addition to commercial computer-aided design (CAD) tools utilized in generic engineering domains, custom design research platforms have been developed for design studies in specific domains, such as truss structures (McComb et al., 2017a), desalination systems (Yu et al., 2016a), cooling systems (McComb et al., 2017b), and solar energy systems (Rahman et al., 2019). However, these existing design research platforms are single domain focused and have addressed only configuration design, leaving systems-level concerns, such as operations and business strategy, untouched. Moreover, the incorporation of intelligent agents in design research platforms is also lacking, limiting the potential for hybrid design team studies. Therefore, the development of collaborative design research platforms is challenging but necessary to advance the research capabilities of the design community.

Towards addressing that gap, this research reviews the currently available design research platforms and identifies four key attributes of a good design research platform. On this basis, we introduce a novel platform for studying hybrid design teams. The design focus of this platform is the configuration and development of unmanned aerial vehicles (UAVs). Distinct from the platforms reviewed, our platform goes beyond pure configurational design by incorporating an operational module and a business strategy module to evaluate the performance of UAV designs within a broader design context subject to scenario development, mission parameters, and multi-person engagement. Moreover, the platform also facilitates the creation and integration of intelligent agents to form hybrid design teams. Flexible design experimentation is supported by our platform across a wide range of studies, which may address variables such as performance, behaviour, interactions, and composition of the hybrid teams.

The remainder of this paper is organized as follows. Section 2 reviews the existing design research platforms, related intelligent agents, and the design studies enabled by the platforms. Next, section 3 presents the four key attributes of a good research platform. Section 4 introduces the design and structure of the UAV design research platform. Finally, section 5 concludes this research and suggests future research directions.

\section{Background}

In this section, the existing literature on design research platforms is reviewed, including the platforms themselves, related intelligent agents, and the design studies conducted through the platforms. Finally, we summarize the research gaps identified from the literature.

\subsection{Existing design research platforms}

Design research platforms typically take advantage of computers to facilitate work on various design tasks, such as 3D modelling, simulation, visualization, and optimization. Commercial CAD tools, such as Autodesk, Siemens NX, and FreeCAD, are amongst the earliest platforms for design research and have recently been employed to study behaviours of designers through recorded operation data (Gopsill et al., 2016; Jin and Ishino, 2006; Mahan et al., 2019; Sivanathan et al., 2015). Design research platforms have also been developed for highly specific design tasks. For example, a graphical user interface was developed by Egan et al. (2015) for myosin design. Likewise, Yu et al. (2016) built a platform to design desalination systems, and McComb et al. (2015a, 2017c) constructed platforms for truss structure and residential cooling system design. Energy3D is a CAD-based research platform for solar energy system design, which also supports economic analysis and evaluation (Rahman et al., 2019; Xie et al., 2018). All of these platforms support performance simulations as one way to assess the quality of new designs and also provide fine-grained data logs that enable detailed analysis of the actions of participants. Three of them (McComb et al., 2015a, 2017c; Xie et al., 2018) support the design process for both individual designers and design teams.

In another strand of research, virtual worlds and virtual reality (VR) technologies are used for developing design research platforms that facilitate human-computer interactions and collaborations in design. Among them, commercial virtual worlds platforms, such as Second Life and Active Worlds, are employed to facilitate and study the processes of human-human and human-computer interactions and collaborations in hybrid design teams (Gül and Maher, 2006; Merrick et al., 2011). Meanwhile, custom VR-based platforms have been constructed to facilitate and study design for assembly (Iglesias et al., 2006; Ritchie et al., 2008; Seth et al., 2005). 


\subsection{Developing intelligent agents}

With advances in computational power and machine learning algorithms, the field of engineering design has seen a wave of interest in intelligent agents. Programmed with sophisticated cognitive logic, intelligent agents can simulate, support, complement, and enhance human design activities. In the development of intelligent agents, defining their dynamic interaction and collaboration with humans is critical. The division of labour in hybrid teams involves not only task allocation but also interaction and mutual support between computers and humans (Madni and Madni, 2018). Parasuraman et al. (2000) proposed a model that categorizes interaction between humans and agents according to the types (information acquisition, information analysis, decision selection, and action implementation) and levels of automation. Similarly, Neef (2006) emphasized two dimensions, coordination type (i.e., standardized coordination, direct supervision, and joint coordination (Mintzberg, 1989)) and collaboration type (i.e., taskwork-oriented and teamwork-oriented), to classify the collaboration in hybrid teams. By formalizing various types of collaboration, intelligent agents can take on different roles in different systems. For example, design agents commonly take on the role of instructing designers, managing pools of existing design knowledge and interact with human designers to provide relevant design knowledge. Kumar et al. (2010) developed a tutor, Avis, to communicate relevant theoretical knowledge with designers through text conversations. More advanced agents can learn relevant design knowledge themselves and then share what they have learned with human designers (Hu and Taylor, 2016).

Another research category consists of computational design agents that, through mimicking cognitive processes, can independently understand and explore given design spaces. For instance, several computational agents have been designed to generate creative and adaptive behaviour through machine learning (Maher et al., 2008; Merrick et al., 2008; Soria Zurita et al., 2018). Additionally, a variety of design agents have been developed to search design spaces using strategies inspired by the cognitive process of human design teams, simulating their behaviour (Lapp et al., 2019; McComb et al., 2015b, 2016). Two design agents were constructed on top of Energy3D to scaffold divergent and convergent design processes, respectively, both of which were powered by genetic algorithms (Schimpf et al., 2018). Furthermore, generative design agents have been developed to produce design alternatives utilizing Generative Adversarial Networks (GANs) (Dering and Tucker, 2017), Recurrent Neural Networks (RNNs) (Stump et al., 2019), and convolutional networks (Dosovitskiy et al., 2017; Raina et al., 2019). A generative design module has also been included in Siemens NX (Haubrock and Bevan, 2017).

\subsection{Relevant design studies}

A wide range of design studies have been enabled by the design research platforms reviewed in Sections 2.1 and 2.2. In particular, data loggers embedded in these platforms collect large quantities of information which enable a variety of data-driven analyses. For example, a stream of design studies conducted sequence analysis on the action data of designers to extract and analyse design process knowledge and heuristics, such as action transitional patterns, hidden design stages, and their correlation with design performance (Bywater et al., 2018; Gopsill et al., 2016; Jin and Ishino, 2006; McComb et al., 2017a, 2017b, 2017d; Ritchie et al., 2008; Schimpf et al., 2018). Other types of analysis are also facilitated through the data captured in existing platforms. McComb et al. (2015a) used data to show that high- and low- performing teams employ different strategies regarding convergence-divergence and simplicity-complexity. Egan et al. (2015) investigated how knowledge of parametric relationships and inter-level causalities conveyed by a design platform influences its users' design proficiency. Sha et al. (2015) studied how behavioural game theory affects multidisciplinary design decision making. In addition, reflective data, such as text recorded in an embedded notepad, was analysed to learn designers' reference knowledge for making design decisions (Goldstein et al., 2015).

Apart from data logging, the capability to simulate human designers or design teams through computational design agents enables a category of team-based design studies and comparison studies. For instance, team design activities were simulated using the CISAT agent-based model, facilitating an examination of the correlation between design performance and team characteristics in terms of the interaction between the optimal team characteristics and problem properties (McComb et al., 2017e). Simulations in the KABOOM agent-based model were used to study various aspects of team composition concerning the cognitive style of members (Lapp et al., 2019). Schimpf et al. (2019) used simulations to 
compare the performance of human designers and computational design agents in convergent and divergent design processes to gain insights into modifying the design agents.

\subsection{Synthesis of gaps}

Several research gaps can be identified through the above literature review. First, many of the design research platforms only focus on configurational design, and broader, yet highly influential, design considerations (such as operations and business strategy) are not addressed. Secondly, although several intelligent agents have been developed, most of them were not developed to support and study hybrid design teams. Figure 1 presents a classification diagram of the design research platforms reviewed here to illustrate these gaps. To this end, more interactive and collaborative intelligent agents are demanded. Finally, more design studies under multiple collaborative design scenarios should be conducted to build an understanding of hybrid design teams (e.g., performance, composition, and dynamics of interactions and collaborations), while few design research platform features have been developed to support and study these complex experimental scenarios.

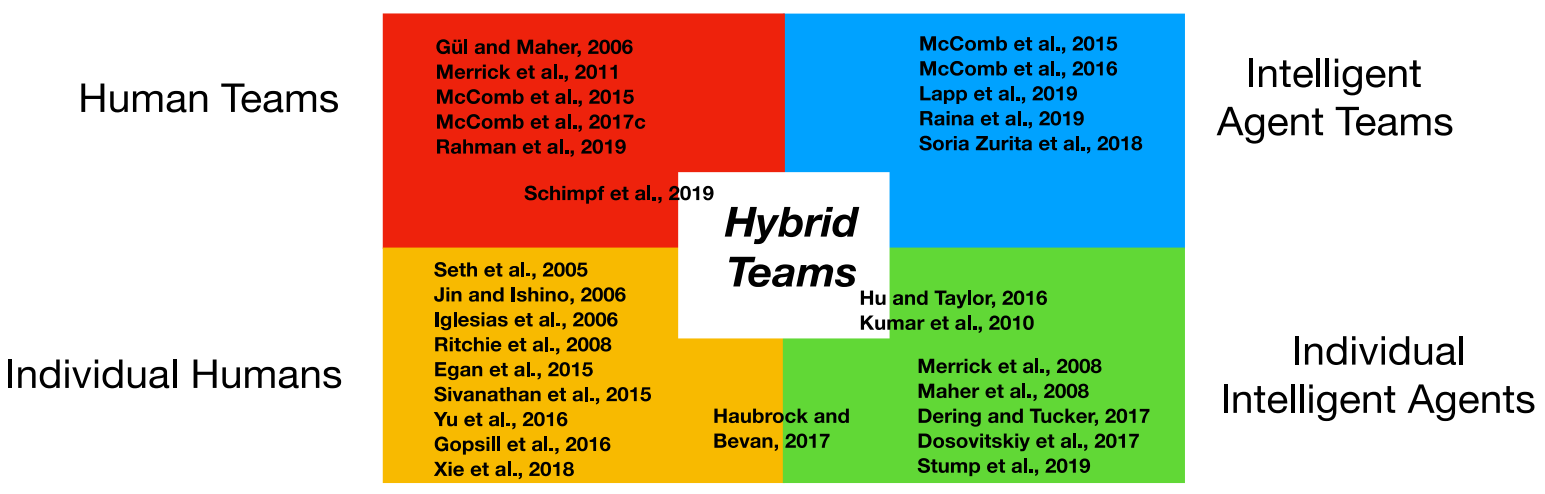

Figure 1. Design research platform classification that lists the reviewed platforms into four categories, with a few making efforts towards hybrid design teams

\section{What makes a good collaborative design research platform?}

The research gaps identified through the literature review pose a common question: what qualifies a good collaborative design research platform for complex system design? This paper identifies four primary attributes that delineate platform utility:

1. Support multi-phase design. The platform should be able to go beyond configurational design to address coupled design considerations from a broader context, such as operational and business plans. This attribute aims to fill the first research gap identified above by integrating coupled design phases, which also empowers researchers to study the influence of the coupling on complex system design.

2. Support hybrid design teams. The platform should include intelligent agents capable of collaborating and interacting with human designers to complete challenging tasks to fulfil the design process. The second research gap can be filled by this attribute, and hybrid design teams can be formed with the incorporated intelligent agents. On this basis, various design studies can be conducted on the interaction and collaboration dynamics of hybrid teams, laying the foundation for addressing the third research gap.

3. Support multiple collaborative design scenarios. Different Application Program Interfaces (APIs) should be developed to allow the platform to support distinct design scenarios, such as purely human teams, hybrid design teams, and purely intelligent agent teams. This attribute aims to address the third research gap by enabling design studies on how hybrid design teams differ from purely human or intelligent agent teams in design process and performance.

4. Support detailed data recording. The platform should be able to compile and export detailed data regarding the design process and design outcomes. This attribute supports various data-driven analyses underlying all the design studies enabled by the first three attributes, also serving as a basis for addressing the third research gap. 


\section{Collaborative design research platform for UAVs}

In this section, we introduce a collaborative design research platform focusing on UAV fleet design. The platform provides a perfect context for studying coupled design phases and human-computer hybrid teaming because it involves a complex multi-phase design process but a limited number of solution components. Using the UAV design research platform as an example, we demonstrate how the four attributes listed above can be embodied. The task entails the design of a UAV fleet for parcel delivery under competition with another delivery company.

\subsection{Three design modules support multiple design phases}

The proposed design research platform is comprised of separate modules for configurational design, operational design, and business planning. The integrated platform provides a comprehensive and seamless environment for UAV design. Figure 2 illustrates the overall architecture of our UAV design research platform. Within the platform, relevant design data can be easily synchronized and utilized across modules to facilitate the coupled design process and decision making of design teams. The crucial data and information flows between the three modules are shown in Figure 2.

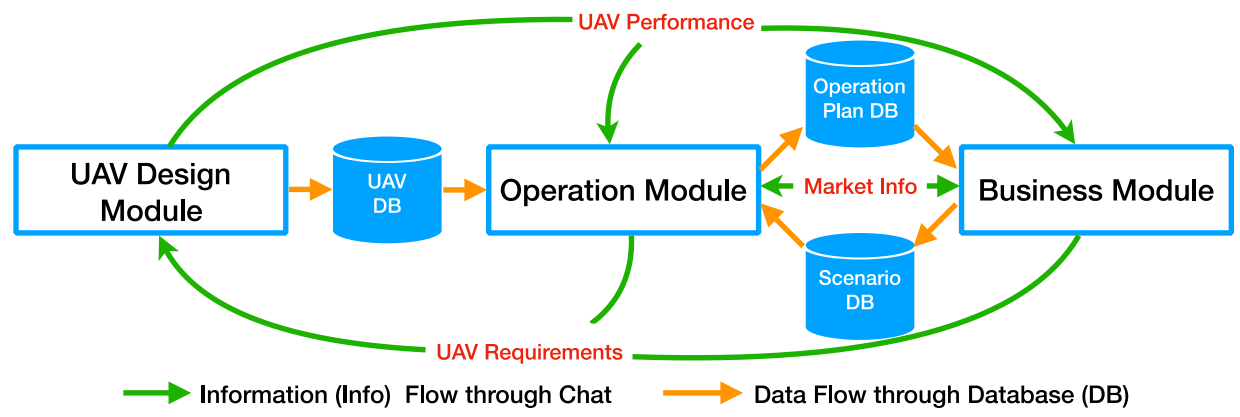

Figure 2. Architecture of the platform with data and information flows between modules

\subsubsection{Configurational design module}

The UAV configurational design interface is shown in Figure 3. Within the interface, a basic initial design is provided from which designers can construct new UAVs by changing component sizes, adding new components, or removing existing components. A component pool is available which includes predesigned components such as motor-rotor pairs, connecting rods, batteries, and foils. The configuration of the constructed UAV is presented in the centre of the interface.

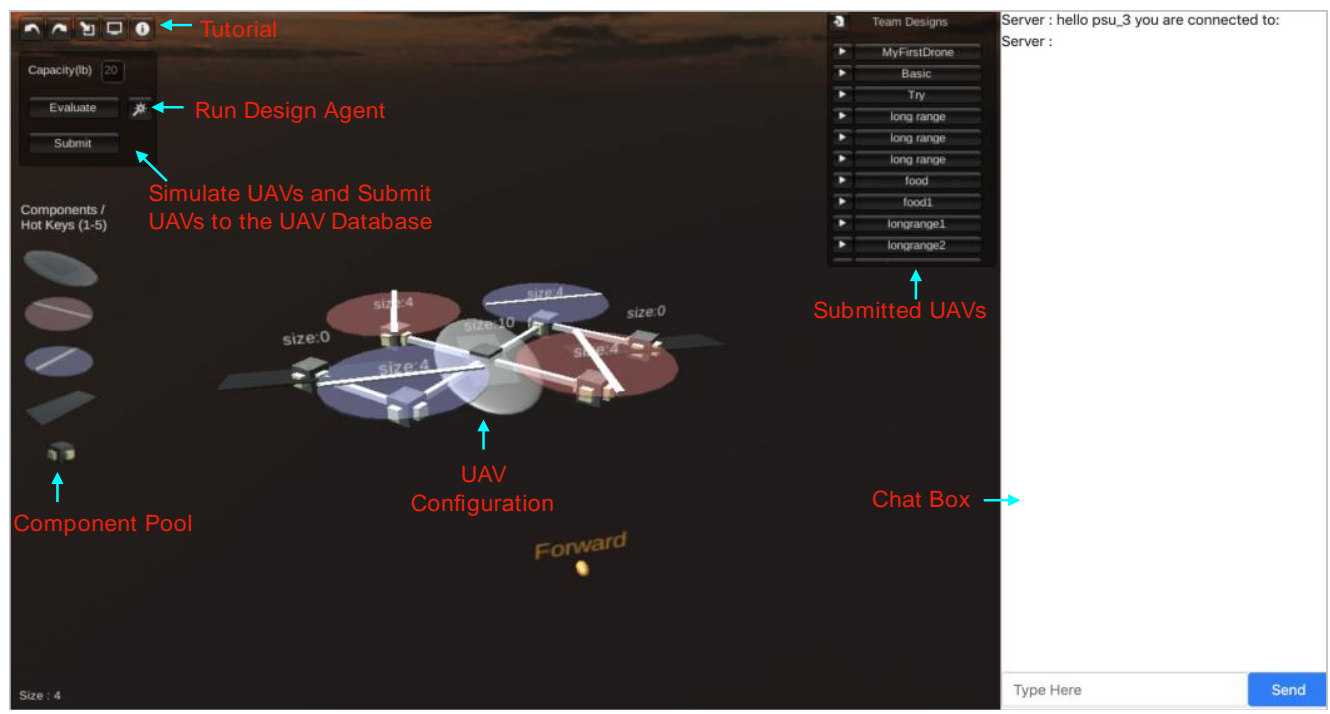

Figure 3. Prototype of UAV configurational design interface 
Further, designers can simulate the validity and the performance (e.g., cost, flying range, and velocity with given payload) of a UAV design. A design agent can be called to generate new UAVs based on a valid UAV design. The simulated designs can be tagged and submitted to a database to facilitate sharing with other team members. A tutorial is embedded which provides an instruction manual for the interface. Moreover, a text-based chat box is included through which designers can communicate design information to other members of the team.

\subsubsection{Operational design module}

The interface of the operational design module is exhibited in Figure 4. The objective of operational design is to develop an operation plan, consisting of a parcel grouping, trip arrangement, and parcel delivery order. To generate the operation plan, two categories of inputs need to be loaded into the interface: (1) UAVs from the UAV database submitted by UAV designers; (2) business scenarios from a scenario database submitted by business planners. A business scenario is visualized by an interactive 3D virtual map, showing the locations of the warehouses, customers, parcel types, and parcel weights. Operation planners need to select suitable UAVs to build a fleet and plan a route for each selected UAV by clicking on the map to construct a complete operation plan. They can also call an operations agent to generate candidate operation plans. An operation plan is automatically evaluated, returning the number of customers being served, the weights being delivered, start-up cost (cost of selected UAVs), operating cost, and the final profit. Each operation plan can be tagged and submitted to an operation plan database to facilitate direct sharing with team members. A tutorial and chat box are also available to facilitate natural language communication.

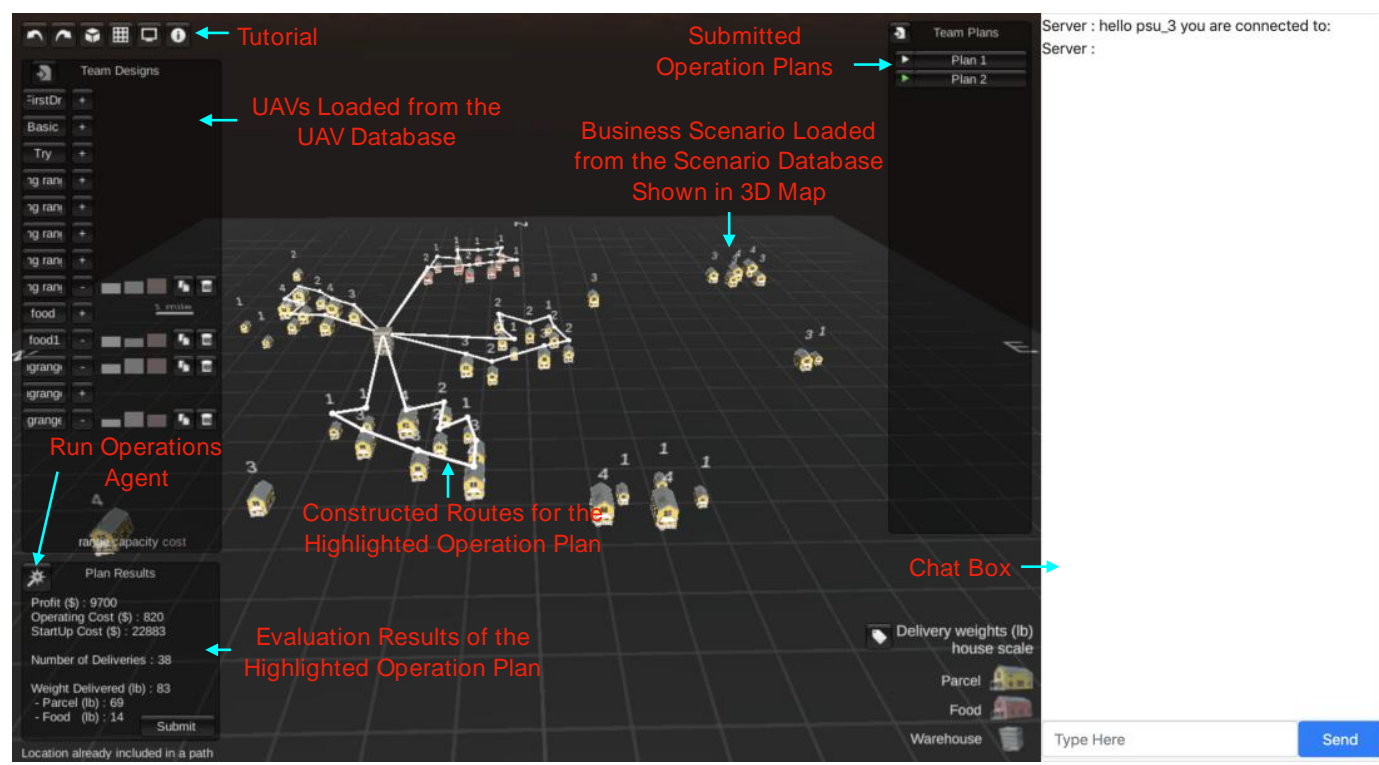

Figure 4. Prototype of UAV operational design and business strategy interface

\subsubsection{Business strategy module}

An interface has also been developed for market planning, which is similar to the interface for operation planning, but containing slightly different functions. In our design scenario, a company provides a "last-mile" parcel delivery service that brings parcels from a nearby warehouse to customer locations. A market plan includes how many and which types of customers the company should serve, as well as how to adapt its business strategy based on the estimated market trend. Based on their knowledge of the market and the capabilities of the company (e.g., number of usable UAVs, flying ranges and speeds and carriable payloads of the UAVs), business planners should create business scenarios by selecting a part of the potential customers as targets that they want to serve. The created scenarios can be submitted to the scenario database, which can be loaded into the operational design module. Business planners can also load operation plans from the operation plan database to learn the change in company capability and modify the business scenario accordingly. At the end of the design process, a business planner should select one operation plan as the final output of their team. 


\subsection{Intelligent agents supporting hybrid design teams}

As mentioned above, two intelligent agents have been developed and incorporated in this UAV design research platform enabling the formation of hybrid teams. The agents each automate a specific task and can be called by designers when they need help to tackle the complexity of the task. For example, a design agent is available in the UAV design module. It can automatically generate UAV designs based on a valid design. Figure 5 demonstrates the design results of the agent, from which human designers can select UAVs as per their demand. The generative design process is realized through training of RNNs on UAV designs represented using a string grammar. The grammar defines all UAV features like the configuration layout, component types, and component sizes (Stump et al., 2019). An operations agent is also available in the operation module. By formulating all the constraints on range, capacity, speed and time in the delivery problem, the operations agent is able to generate and optimize candidate operation plans through running linear programming. Besides these two intelligent agents, it is possible to generate a variety of other agents to automate different testbed tasks.

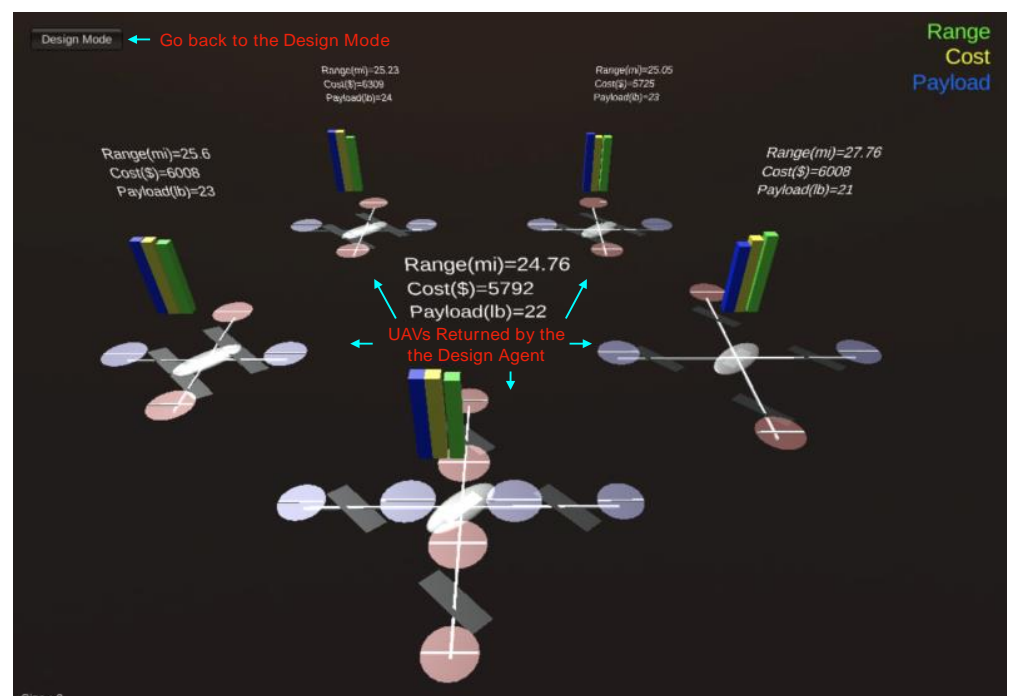

Figure 5. UAVs designed by the design agent

\subsection{Distinct APIs supporting multiple experimental scenarios}

Built on the modular platform and well-defined intermediate databases, distinct APIs have been developed for manipulating the UAV collaborative design platform to support multiple experimental scenarios. By enabling or disabling the access of humans and intelligent agents to the corresponding interfaces and databases through different APIs, the platform can support purely human teams, hybrid teams, and purely intelligent agent teams, respectively. This opens up a flexible environment for developing and experimenting with various computational design agents.

\subsection{Logger supporting detailed data recording}

Since the analysis of audio and video data that record the process of designers working is challenging and increases privacy concerns, this design research platform is designed to focus on the behaviourbased data recorded by the embedded data logger in a non-intrusive manner. In each of three modules, a data $\log$ records every action of designers for developing a UAV, an operation plan, or a market plan, including the calling of the two intelligent agents and their design results. Every piece of information is timestamped. The data logs can be exported as JavaScript Object Notation (JSON) files. The chat texts among teammates are also recorded and timestamped. The collected data can potentially support many of the design studies presented in the literature review. Meanwhile, the integration of the three design modules enables studies that investigate the influence of the coupling between different design phases on the design process and outcomes. The incorporation of the intelligent agents directly facilitates studies on how designers perceive, interact and collaborate with the intelligent agents and how these factors influence design performance. The multiple design 
scenarios enable a variety of comparison studies such as how hybrid teams differ from human or intelligent agent teams.

\section{Conclusion}

With the increasing prevalence of intelligent agents that assist with the design of complex engineered systems, there is a need to better understand the behaviour of hybrid human-computer teams. Improved collaborative design research platforms are necessary to enable experiments to investigate these hybrid teams. In this paper, the current state of the art about collaborative design research platforms was reviewed. It was identified that although several design research platforms have been developed, few of them support integrated design beyond configuration alone, and few incorporate intelligent agents that support hybrid design team studies. On this basis, we identified four attributes that qualify a good collaborative design research platform: (1) support multiple design phases to address a broader design context, (2) support hybrid teams with intelligent agents, (3) support multiple design scenarios according to experiment requirements, and (4) support data logging to enable various data-driven analyses.

Here we introduced a novel UAV design research platform to demonstrate how to embody these attributes. The platform integrates configurational design, operational design and business planning of a fleet of UAVs contextualized within parcel delivery tasks. Intelligent agents are incorporated in the platform that can interact with designers and take design tasks as team members to enable the formation of human-computer hybrid design teams. The platform can be manipulated through different APIs to support distinct design scenarios, such as purely human teams, hybrid teams, and purely intelligent agent teams. A data logger allows the platform to record the design process regarding actions and progress, supporting the data-driven design studies on coupled design phases, hybrid design teams, and comparison between multiple experimental scenarios.

Moreover, the platform is designed to support design research in several ways: (1) the UAV design is based on predefined components, which simplifies the design tasks and benefits design studies by allowing designers to learn more easily and quickly during the design process; (2) The text-based communication among designers provides cleaner and more interpretable data for studies on design communication; (3) multiple APIs support different experimental scenarios; (4) fine-grained data logs provide rich opportunities for studying the design process of hybrid design teams. However, this work is still an initial foray into the development of a design research platform. Ultimately, the platform will empower design researchers to engage in a variety of research endeavours, including the integration of coupled design processes, hybrid teaming, trust in intelligent agents, advanced design automation, among other tasks. Future work may explore each of these capabilities through flexible design experimentation. Research questions concerning the perception and behaviour of human designers during interaction with intelligent agents and their influence on design performance may also be addressed.

\section{Acknowledgement}

This material is based upon work supported by the Defense Advanced Research Projects Agency through cooperative agreement N66001-17-1-4064. Any opinions, findings, and conclusions or recommendations expressed in this paper are those of the authors and do not necessarily reflect the views of the sponsors.

\section{References}

Bywater, J.P. et al. (2018), "Using machine learning techniques to capture engineering design behaviors", Proceedings of International Conference of the Learning Sciences, ICLS, Vol. 3, pp. 1359-1360. https://doi.org/10.22318/cscl2018.1359

Cooper, S. et al. (2010), "Predicting protein structures with a multiplayer online game", Nature, Vol. 466 No. 7307, pp. 756-760. https://doi.org/10.1038/nature09304

Dering, M.L. and Tucker, C.S. (2017), "Generative adversarial networks for increasing the veracity of big data", 2017 IEEE International Conference on Big Data, IEEE, pp. 2595-2602. https://doi.org/10.1109/ BigData.2017.8258219 
Dosovitskiy, A. et al. (2017), "Learning to Generate Chairs, Tables and Cars with Convolutional Networks", IEEE Transactions on Pattern Analysis and Machine Intelligence, IEEE Computer Society, Vol. 39 No. 4, pp. 692-705. https://doi.org/10.1109/TPAMI.2016.2567384

Egan, P. et al. (2015), "Improving human understanding and design of complex multi-level systems with animation and parametric relationship supports", Design Science, Vol. 1. https://doi.org/10.1017/dsj.2015.3

Goldstein, M.H. et al. (2015), "High school students' ability to balance benefits \& tradeoffs while engineering green buildings", ASEE Annual Conference and Exposition, Seattle, WA. https://doi.org/10.18260/p.24183

Gopsill, J.A. et al. (2016), "Automatic generation of design structure matrices through the evolution of product models", Artificial Intelligence for Engineering Design, Analysis and Manufacturing: AIEDAM, Vol. 30 No. 4, pp. 424-445. https://doi.org/10.1017/S0890060416000391

Gül, L.F. and Maher, M.L. (2006), "Studying design collaboration in DesignWorld: An augmented 3D virtual world", Proceedings - Computer Graphics, Imaging and Visualisation: Techniques and Applications, CGIV'06, Vol. 2006, pp. 471-477. https://doi.org/10.1109/CGIV.2006.82

Haubrock, B. and Bevan, P. (2017), Next Generation Design with NX.

Hu, Y. and Taylor, M. (2016), "Work in progress: A Computer-Aided Design Intelligent Tutoring System Teaching Strategic Flexibility”, 2016 ASEE Annual Conference \& Exposition Proceedings. https://doi.org/ $10.18260 / \mathrm{p} .27208$

Iglesias, R. et al. (2006), “A peer-to-peer architecture for collaborative haptic assembly", Proceedings - IEEE International Symposium on Distributed Simulation and Real-Time Applications, pp. 25-34. https://doi.org/ 10.1109/DS-RT.2006.3

Jin, Y. and Ishino, Y. (2006), "DAKA: Design activity knowledge acquisition through data-mining", International Journal of Production Research, Vol. 44 No. 14, pp. 2813-2837. https://doi.org/10.1080/ 00207540600654533

Kollat, J.B. and Reed, P. (2007), “A framework for Visually Interactive Decision-making and Design using Evolutionary Multi-objective Optimization (VIDEO)”, Environmental Modelling and Software, Vol. 22 No. 12, pp. 1691-1704. https://doi.org/10.1016/j.envsoft.2007.02.001

Kumar, R. et al. (2010), "Socially capable conversational tutors can be effective in collaborative learning situations", Lecture Notes in Computer Science, Vol. 6094, pp. 156-164. https://doi.org/10.1007/978-3-64213388-6_20

Lapp, S., Jablokow, K. and McComb, C. (2019), "KABOOM: an agent-based model for simulating cognitive style in team problem solving", Design Science, Vol. 5, pp. 1-32. https://doi.org/10.1017/dsj.2019.12

Madni, A. and Madni, C. (2018), "Architectural Framework for Exploring Adaptive Human-Machine Teaming Options in Simulated Dynamic Environments”, Systems, MDPI AG, Vol. 6 No. 4, pp. 44. https://doi.org/ $10.3390 /$ systems6040044

Mahan, T. et al. (2019), "Pulling at the digital thread: Exploring the tolerance stack up between automatic procedures and expert strategies in scan to print processes", Journal of Mechanical Design, Vol. 141 No. 2. https://doi.org/10.1115/1.4041927

Maher, M.L., Merrick, K. and Saunders, R. (2008), “Achieving Creative Behaviour Using Curious Learning Agents", AAAI Spring Symposium: Creative Intelligent Systems, pp. 40-46.

McComb, C., Cagan, J. and Kotovsky, K. (2015a), "Rolling with the punches: An examination of team performance in a design task subject to drastic changes", Design Studies, Vol. 36 No. C, pp. 99-121. https://doi.org/10.1016/j.destud.2014.10.001

McComb, C., Cagan, J. and Kotovsky, K. (2015b), "Lifting the Veil: Drawing insights about design teams from a cognitively-inspired computational model", Design Studies, Vol. 40, pp. 119-142. https://doi.org/ 10.1016/j.destud.2015.06.005

McComb, C., Cagan, J. and Kotovsky, K. (2016), "Drawing Inspiration from Human Design Teams for Better Search and Optimization: The Heterogeneous Simulated Annealing Teams Algorithm", Journal of Mechanical Design, Vol. 138 No. 4, pp. 1-6. https://doi.org/10.1115/1.4032810

McComb, C., Cagan, J. and Kotovsky, K. (2017a), "Utilizing Markov Chains to Understand Operation Sequencing in Design Tasks", Design Computing and Cognition '16, pp. 401-418. https://doi.org/10.1007/ 978-3-319-44989-0_22

McComb, C., Cagan, J. and Kotovsky, K. (2017b), "Capturing Human Sequence-Learning Abilities in Configuration Design Tasks Through Markov Chains”, Journal of Mechanical Design, Vol. 139 No. 9, pp. 1-12. https://doi.org/10.1115/1.4037185

McComb, C., Cagan, J. and Kotovsky, K. (2017c), "Data on the configuration design of internet-connected home cooling systems by engineering students", Data in Brief, Vol. 14, pp. 773-776. https://doi.org/ 10.1016/j.dib.2017.08.050 
McComb, C., Cagan, J. and Kotovsky, K. (2017d), "Mining Process Heuristics From Designer Action Data via Hidden Markov Models", Journal of Mechanical Design, Vol. 139 No. 11, pp. 111412. https://doi.org/ $10.1115 / 1.4037308$

McComb, C., Cagan, J. and Kotovsky, K. (2017e), "Optimizing design teams based on problem properties: Computational team simulations and an applied empirical test”, Journal of Mechanical Design, Vol. 139 No. 4. https://doi.org/10.1115/1.4035793

Merrick, K., Maher, M.L. and Saunders, R. (2008), “Achieving adaptable behaviour in intelligent rooms using curious supervised learning agents", CAADRIA 2008 - The Association for Computer-Aided Architectural Design Research in Asia: Beyond Computer-Aided Design, pp. 185-192.

Merrick, K.E., Gu, N. and Wang, X. (2011), "Case studies using multiuser virtual worlds as an innovative platform for collaborative design", Electronic Journal of Information Technology in Construction, Vol. 2011 No. 16, pp. 165-188.

Mintzberg, H. (1989), “The Structuring of Organizations", In: Readings in Strategic Management, Macmillan Education, UK, pp. 322-352. https://doi.org/10.1007/978-1-349-20317-8_23

Neef, M. (2006), "A Taxonomy of human - agent team collaborations", Proceedings of the 18th BeNeLux Conference on Artificial Intelligence (BNAIC 2006), Namur, Belgium.

Parasuraman, R., Sheridan, T.B. and Wickens, C.D. (2000), "A model for types and levels of human interaction with automation", IEEE Transactions on Systems, Man, and Cybernetics Part A:Systems and Humans, Vol. 30 No. 3, pp. 286-297. https://doi.org/10.1109/3468.844354

Rahman, M.H. et al. (2019), “A Computer-Aided Design Based Research Platform for Design Thinking Studies", Journal of Mechanical Design, Vol. 141 No. 12. https://doi.org/10.1115/1.4044395

Raina, A., McComb, C. and Cagan, J. (2019), "Learning to Design From Humans: Imitating Human Designers Through Deep Learning”, Journal of Mechanical Design, Vol. 141 No. 11. https://doi.org/10.1115/1.4044256

Ritchie, J.M. et al. (2008), "The Analysis of Design and Manufacturing Tasks Using Haptic and Immersive VR Some Case Studiess", Product Engineering, pp. 507-522. https://doi.org/10.1007/978-1-4020-8200-9_27

Schimpf, C.T. et al. (2018-June), "Work in progress: A Markov chain method for modeling student behaviors", ASEE Annual Conference and Exposition, Conference Proceedings.

Seth, A., Su, H.J. and Vance, J.M. (2005), "A desktop networked haptic VR interface for mechanical assembly", American Society of Mechanical Engineers, Computers and Information in Engineering Division, CED, Vol. 10, pp. 173-180. https://doi.org/10.1115/IMECE2005-81873

Sha, Z., Kannan, K.N. and Panchal, J.H. (2015), "Behavioral Experimentation and Game Theory in Engineering Systems Design”, Journal of Mechanical Design, Vol. 137 No. 5. https://doi.org/10.1115/1.4029767

Simpson, T.W. et al. (2011), "Trade Space Exploration: Assessing the Benefits of Putting Designers 'Back-inthe-Loop' during Engineering Optimization”, In: Human-in-the-Loop Simulations, Springer, London, pp. 131-152. https://doi.org/10.1007/978-0-85729-883-6_7

Simpson, T.W. and Martins, J.R.R.A. (2011), "Multidisciplinary design optimization for complex engineered systems: Report from a national science foundation workshop", Journal of Mechanical Design, Vol. 133 No. 10. https://doi.org/10.1115/1.4004465

Sivanathan, A. et al. (2015), "The application of ubiquitous multimodal synchronous data capture in CAD", CAD Computer Aided Design, Vol. 59, pp. 176-191. https://doi.org/10.1016/j.cad.2013.10.001

Soria Zurita, N.F. et al. (2018), "Design of Complex Engineered Systems Using Multi-Agent Coordination", Journal of Computing and Information Science in Engineering, Vol. 18 No. 1. https://doi.org/10.1115/ 1.4038158

Stump, G.M. et al. (2019), "Spatial Grammar-Based Recurrent Neural Network for Design Form and Behavior Optimization", Journal of Mechanical Design, Vol. 141 No. 12, https://doi.org/10.1115/1.4044398

Xie, C. et al. (2018), "Learning and teaching engineering design through modeling and simulation on a CAD platform", Computer Applications in Engineering Education, Vol. 26 No. 4, pp. 824-840. https://doi.org/ $10.1002 /$ cae. 21920

Yu, B.Y. et al. (2016a), "Human behavior and domain knowledge in parameter design of complex systems", Design Studies, Vol. 45, pp. 242-267. https://doi.org/10.1016/j.destud.2016.04.005

Zhang, X. et al. (2012), "Supporting knowledge exploration and discovery in multi-dimensional data with interactive multiscale visualisation", Journal of Engineering Design, Vol. 23 No. 1, pp. 23-47. https://doi.org/10.1080/09544828.2010.487260 